\title{
Flower-like copper sulfide nanocrystals are highly effective against chloroquine- resistant plasmodium falciparum and the malaria vector Anopheles stephensi
}

\begin{abstract}
Anopheles stephensi is a mosquito of outstanding public health relevance, acting as a major vector of malaria in a number of tropical and subtropical areas worldwide. In recent years, important efforts have been conducted to propose nano-formulated larvicides as valuable alternatives to synthetic insecticides currently marketed. In the present study, the toxicity of flower-like copper sulfide (CuS) nanocrystals has been investigated on the malaria vector A. stephensi and Plasmodium parasites. Characterization of synthesized CuS nanocrystals was carried out using FTIR spectroscopy, XRD analysis, FESEM, HR-TEM and EDS. In mosquitocidal assays, $\mathrm{LC}_{50}$ values ranged from $23.347 \mathrm{ppm}$ (first-instar larvae) to $48.789 \mathrm{ppm}$ (pupae). In vitro anti-plasmodial activity of $\mathrm{CuS}$ nanoflowers was evaluated against chloroquine-resistant (CQ-r) and CQ-sensitive (CQ-s) strains of Plasmodium falciparum. $\mathrm{IC}_{50}$ were $83.44 \mu \mathrm{g} / \mathrm{mL}$ (CQ-s) and $87.15 \mu \mathrm{g} / \mathrm{mL}$ (CQ-r). However, in vivo antiplasmodial experiments conducted on Plasmodium berghei infecting albino mice showed limited activity of $\mathrm{CuS}$ nanocrystals, if compared to CQ. Overall, our findings showed that chemically synthesized flower-like $\mathrm{CuS}$ nanocrystals are promising to improve the effectiveness of mosquito control programs, as well as to develop novel antiplasmodial drugs.
\end{abstract}

Keyword: Anopheline; Antiplasmodial activity; Chloroquine; Plasmodium berghei 